\section{Checkliste Traumatologie}

— Die erste Auflage der "Checkliste Traumatologie" erschien 1981 und hat mir als damals jungem Assistenzarzt wertvolle Dienste geleistet. Rund 35 Jahre später liegt die nun 8. Auflage vor, wobei das Buch mit den Jahren zwar erheblich an Umfang zugenommen, dabei aber seine gute Strukturierung nicht verloren hat. In 39 Kapiteln gibt das Werk einen hervorragenden Überblick über die gesamte Traumatologie und ist auf insgesamt 39 Kapitel angewachsen. Zusammen mit ihrem Pendant, der "Checkliste Orthopädie", bildet sie das große Fachgebiet Orthopädie und Unfallchirurgie komplett ab.

Ein kapitelübergreifendes Farbleitsystem erleichtert die schnelle Orientierung. Die in einzelnen Kapiteln dargestellten Krankheiten wurden einer strikten Gliederung unterzogen: Grundlagen, klinische Symptomatik, diagnostisches Vorgehen, Therapieprinzipien, konservative Therapie, operative Therapie, Nachbehandlung und Prognose. Dies ermöglicht dem Leser beim Nachschlagen eine schnelle Übersicht. Komplikationen und deren Behandlungen wurde ebenso ein eigenes Kapitel gewidmet wie Verletzungen bei Kindern. Fazit: Auch in der 8. Auflage ist die "Checkliste Traumatologie" neben der "Checkliste Orthopädie" ein Muss für den Arzt in der Weiterbildung zum Facharzt für Orthopädie und Unfallchirurgie, aber auch ein hoch empfehlenswertes Nachschlagewerk für "alte Hasen“. Das Preis-Leistungs-Verhältnis darf als hervorragend bezeichnet werden.

Prof. Dr. med. Klaus M. Peters, Nümbrecht

\section{Checkliste Traumatologie}

Bühren V., Keel M., Marzi I.

8. Auflage, Thieme-Verlag 2016, 656 Seiten, 580 Abbildungen einschließlich E-

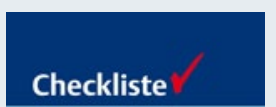
Book; ISBN 978-3$13-5981086 ; 49,99 €$
Eintrittstore aber oft der Adobe Flash Player und eben die bereits erwähnten E-Mail-Anhänge sind, können auch Besitzer von Apple-Rechnern betroffen sein.

\section{Einbußen im fünfstelligen Bereich}

Die Erpresser präsentieren sich „dreist und frech", wie der internistische Kollege weiter erzählte, mit einem auf der Festplatte abgelegten Schreiben. Gegen Bezahlung in Bitcoins - einer digitalen Währung - würde die Freischaltung der verschlüsselten Daten veranlasst. Doch weil es keine Garantie dafür gibt, dass die Daten anschließend auch tatsächlich entschlüsselt werden, hatte sich zumindest der Internist dazu entschlossen, nicht zu zahlen. Der Plan: Das Team wollte die Praxisdaten selbst rekonstruieren. „Was selbstverständlich mit einem erheblichen finanziellen und zeitlichen Aufwand verbunden ist. Hinzu kommt der Umsatzausfall für circa drei geschlossene Praxistage - ohne EDV können moderne Praxen nicht mehr betrieben werden. Hier sind schnell fünfstellige Einbußen erreicht", berichtete der Internist.

\section{Datensicherung - und zwar mit Mehrfachschutz}

Das Stichwort lautet Datensicherung. Dazu rät auch das BSI eindringlich, gerade wegen der aktuell gehäuften Fälle von Ransomware-Attacken auf kleinere Betriebe und Privatpersonen. Im letzten Jahr hat es aber auch das System eines Klinikkonsortiums getroffen. Hier hatte "Cryptowall“ zugeschlagen. Weil in großen Kliniken die Datensicherung meist ein Standardprozess ist, konnte der Datenausfall jedoch auf zwölf Stunden begrenzt werden. Aber: Ein weiterer finanzieller Schaden sei nicht ausgeschlossen, heißt es, da Abrechnungen unter Umständen nicht mehr nachvollzogen werden könnten.

Dabei sind die Spielregeln für die Datensicherung einfach:

_ Sie sollte regelmäßig (je nach Praxisgröße täglich bis wöchentlich) auf einem externen Speichermedium vorgenommen werden. Hierzu eignen sich etwa gute RAID(redundant array of independent disks)-Systeme. Dahinter verbirgt sich ein Verbund un- abhängiger Festplatten in einem Gehäuse. Die Daten werden beim Speichern gleichzeitig auf mehreren Festplatten abgelegt. Das senkt das Risiko, dass sie in irgendeiner Form nicht auslesbar sind. In kleinen Praxen kann aber auch eine normale USBFestplatte für die Datensicherung genutzt werden.

_Die Datensicherung sollte immer getrennt vom Rechnersystem der Praxis aufbewahrt und vor allem nicht dauerhaft an dieses angeschlossen werden, denn: „Viele Verschlüsselungstrojaner können auch Daten auf externen und Netzwerklaufwerken unbrauchbar machen“, mahnt das BSI. -Außerdem sollte das Praxisteam anhand einiger ausgewählter Dateien prüfen, ob sich die gesicherten Dateien tatsächlich wiederherstellen lassen und die Datensicherung funktioniert.

\section{Am besten das ganze Programm}

Zusätzlich benötigen Praxen diverse Schutzmechanismen, die Eindringlinge von vornherein abwehren. Dazu zählen in jedem Fall eine aktivierte, aktuelle Firewall (die meisten Internetrouter haben bereits eine integrierte Firewall), ein aktuell gehaltener Antivirenscanner sowie ein Betriebssystem und einen Internetbrowser, die durch regelmäßige Updates dem neuesten Sicherheitsstandard entsprechen. Wichtig ist laut BSI darüber hinaus eine gesunde Portion Misstrauen gegenüber unbekannten EMail-Absendern, etwa mit Rechnungsanhang.

Gleiche Vorsicht gelte bei E-Mail-Faxen. Beim Surfen im Internet hilft es, wenn der Virenscanner sichere und unsichere Websites symbolisch kennzeichnet. Praxen müssen außerdem die Schweigepflicht auch für die Patientendaten auf den Rechnern beachten. Es gilt daher, die Systeme - falls der Nachweis doch einmal strafrechtlich nötig ist - nach bestem Wissen durch den aktuellen Stand der Technik zu schützen.

Der eingangs erwähnte Internist geht mittlerweile sogar noch einen Schritt weiter: „Der Mailrechner wird vom Netz und vom Server abgehängt; nur dort lokal geprüfte Mails werden weiter geleitet."

Rebekka Höhl 